\title{
Virtual bird's-eye for remote operation of unmanned construction machinery
}

\author{
Noritaka Sato, Akihiro Fukuda* \\ Morita and Sato Laboratory, Department of Electrical and Mechanical Engineering, Nagoya Institute of Technology, \\ Gokiso-cho, Showa-ku, Nagoya, Aichi, 466-8555 Japan \\ Email:a.fukuda.975@nitech.ac.jp \\ http://watt.web.nitech.ac.jp/index_2.html
}

\begin{abstract}
Recently, remote operation of construction machinery is required to release humans from dangerous places. However, the risk of collisions and operation time increases because of lacking a sense of distance. To solve those problems, we propose a method to display a virtual bird's-eye view. Experiments are carried out to verify the effectiveness of the proposed method which can reduce the number of collisions and operation time comparing to the conventional method.
\end{abstract}

Keywords: virtual reality, remote operation, construction machinery, UI

\section{Introduction}

Recently, remote operation of construction machinery is required to release humans from dangerous places. One of the situations where the remote operation of construction machinery is required is "the sorting operation of slags" in iron factories shown in Fig. 1. "The sorting operation of slags" is the work of sieving slags with a power shovel equipped with a bucket and consists of the following three processes: shoveling up slags, sieving slags, and releasing the remains. The workplace is harsh for workers because of flying dust, the high temperature, and the high humidity.

The operation by the remote control of a construction machine was carried out by using the active robot $\mathrm{SAM}^{1}$ in the past. However, problems such as a significant increase in working time and the collision of the power shovel with obstacles have occurred. It is considered that the reason for them is lacking the sense of distance because of only using a monocular camera.

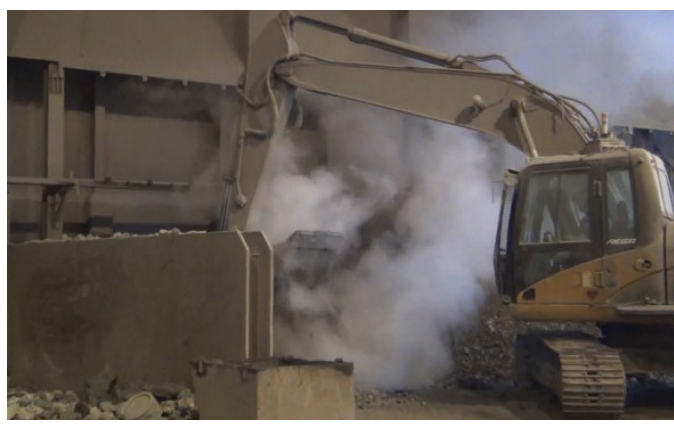

Fig. 1 The sorting operation of slags

The research by Shiroma et al. has proved that a bird's-eye view of the operation target improves the sense of distance ${ }^{2}$. However, it is difficult to install cameras in the workplace and take bird's-eye view images of the construction machine due to problems such as the enormous amount of data communication, limited installation position, and poor visibility due to dust. The researchers also proposed a system that creates a virtual 
bird's-eye view by superimposing a CG model of the robot on an image obtained while the robot is moving ${ }^{3}$.

In the previous paper, we proposed a new method to support the remote operation of construction machinery based on the system which creates virtual bird's-eye view images and verified the effectiveness of it on the sorting operation of slags in the real environment with a professional operator ${ }^{4}$. However, there was a problem which was an insufficient number of trials as it was hard to have the professional operator to make time. In this paper, we did an additional experiment to get confirmation of the effectiveness of the proposed system.

\section{Proposed system}

\subsection{System overview}

In the proposed system, virtual bird's-eye view images are created by superimposing a CG of the power shovel on images. Since the position of the power shovel did not change during the operation, the position and the posture of the CG without the posture of the body of the power shovel are changed based on measured values obtained from sensors attached to the power shovel.

\subsection{Real-time video}

In addition to virtual bird's-eye view images, realtime videos are displayed. In the experiment using the actual power shovel, cameras are mounted as shown in Fig. 2 for displaying slags and the inside of the bucket during sieving.

In the experiment in this study, a camera for slags shown in Fig. 2 is used, because the motion of the sieving is not required in the experiment.

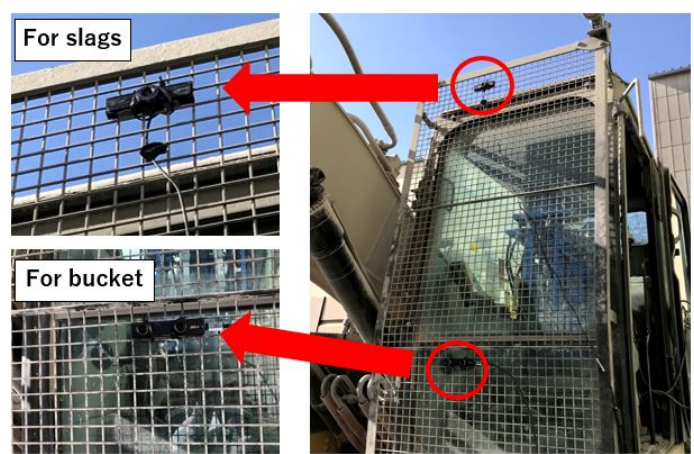

Fig. 2 Cameras on the power shovel

\subsection{Sensing position and posture}

To sense the position and the posture of the power shovel, we mounted some sensors on it. Fig. 3 shows mounted sensors. The angle of each link is calculated from the acceleration measured by the IMU attached to them. The position and the posture of the power shovel are measured by taking a picture of the AR markers and using ARToolKit ${ }^{3}$ to get the relative position and posture from the external camera.

In the experiment in this study, the angle of each link is measured by an encoder on the servo motor, and the position and the posture of the body are fixed, therefore there is no sensor to measure them.
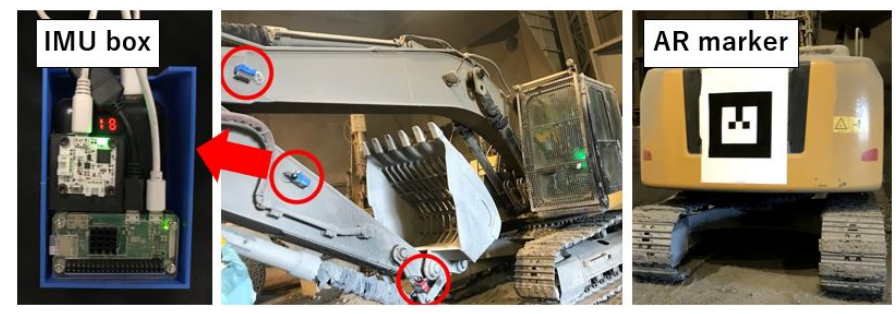

Fig. 3 Installed sensors

\subsection{Creating virtual bird's-eye view images}

Virtual bird's-eye view images were created using a game engine, Unity by the following steps in shown Fig. 4:

(i) Putting the images taken in advance inside the virtual space.

(ii) Putting a 3D CG model of the power shovel inside the virtual space based on its measured position and posture.

(iii) Getting a view from the position where the image was taken. (The CG model is virtually superimposed)

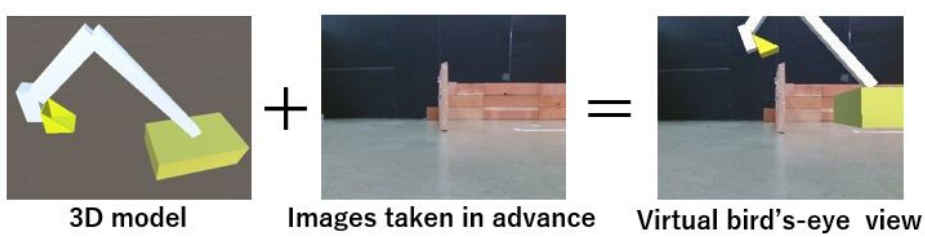

Fig. 4 Generating virtual bird's-eye view 


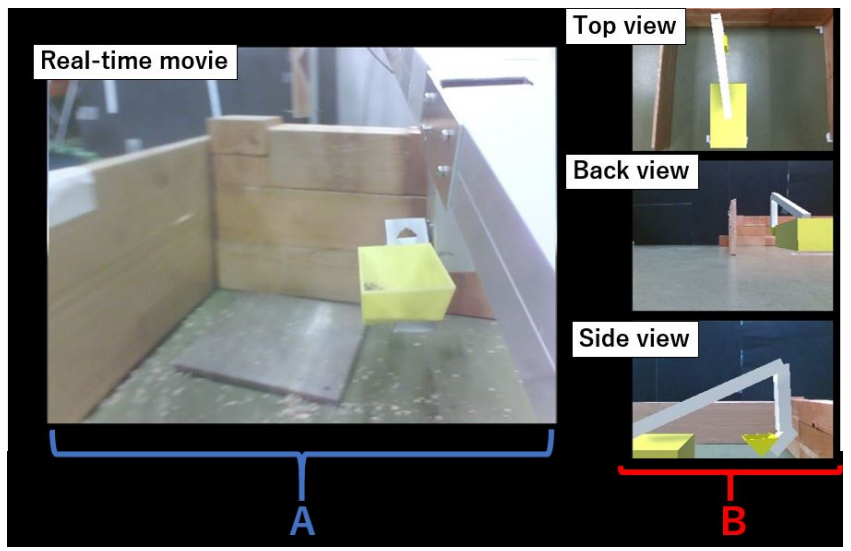

Fig. 5 The image shown to operators

Fig. 5 shows the video displayed to the operator. The left-side video (A) is a real-time video and the right-side videos (B) are virtual bird's-eye view images.

\section{Experiments}

\subsection{Experimental details}

The simulation was conducted in the environment and procedure shown in Fig. 6. Two male subjects in their 20s have no operation experience in the robot and were given 10 minutes to practice. The sorting operations were conducted 10 times each using the real-time video only ("A" shown in Fig. 5) and the proposed system ("A" and "B" shown in Fig. 5). They were conducted in an alternating cycle of five times to remove the effect of the order.

The data recorded were operation time, number of collisions, and subjective evaluation by questionnaire.

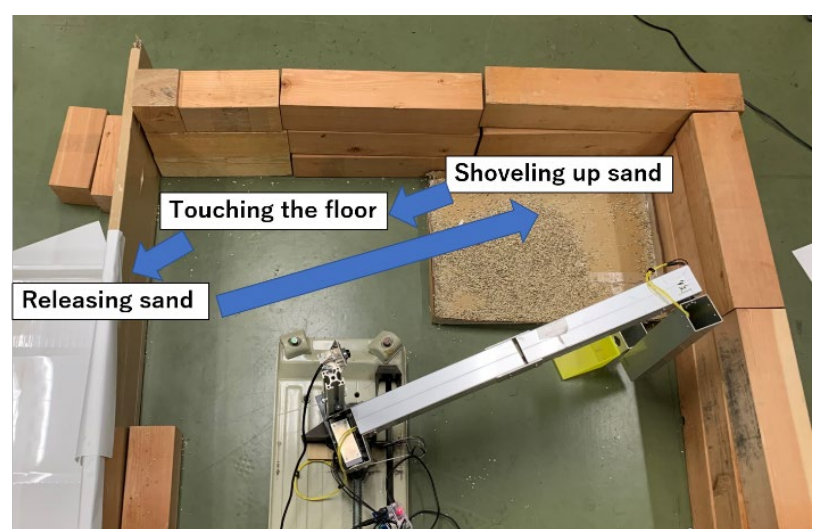

Fig. 6 The environment of the simulation

\subsection{Result}

Figs. 7, 8 show each subject's results: average operation time of 10 times and standard deviation. In subject 1 , an average operation time was $53.7 \mathrm{sec}$ when using the only real-time video and $48.4 \mathrm{sec}$ when using the proposed system, and it was reduced by $9.87 \%$. The standard deviation was $9.58 \mathrm{sec}$ when using the real-time video and $7.39 \mathrm{sec}$ when using the proposed system, and it was reduced by $22.8 \%$. In subject 2 , the average operation time was $46.7 \mathrm{sec}$ when using the real-time video and $39.1 \mathrm{sec}$ when using the proposed system, and it was reduced by $16.3 \%$. The standard deviation was $12.0 \mathrm{sec}$ when using the real-time video and $6.10 \mathrm{sec}$ when using the proposed system, and it was reduced by $49.1 \%$.

On the other hand, an average of the number of collisions was 0.36 times per one cycle when using the real-time video and 0.16 times per one cycle when using the proposed system in subject 1. 0.23 times per one cycle when using the real-time video and 0 times per one cycle when using the proposed system in subject 2 .

In the questionnaire, the usability of the virtual bird's-eye view images was surveyed. Subject 1 answered that the side view and the top view were useful to recognize the sense of distance between the bucket and walls and the back view was not useful. On the other hand, subject 2 answered that only the side view was useful.

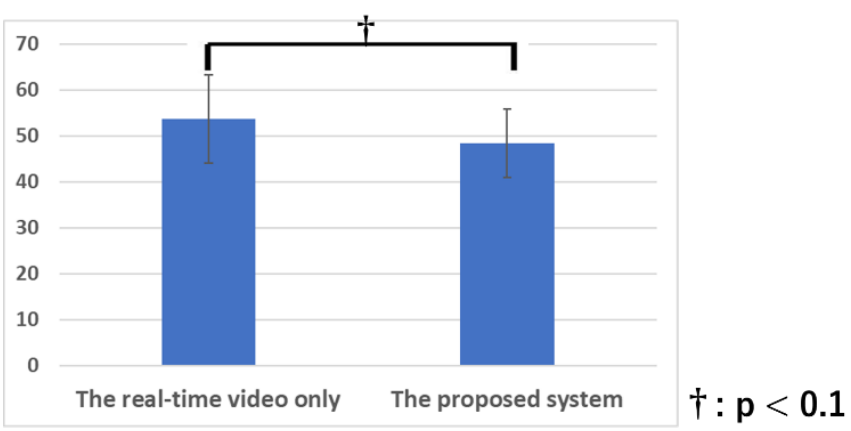

Fig. 7 Operation time (Subject1)

(C) The 2021 International Conference on Artificial Life and Robotics (ICAROB2021), January 21 to 24, 2021 


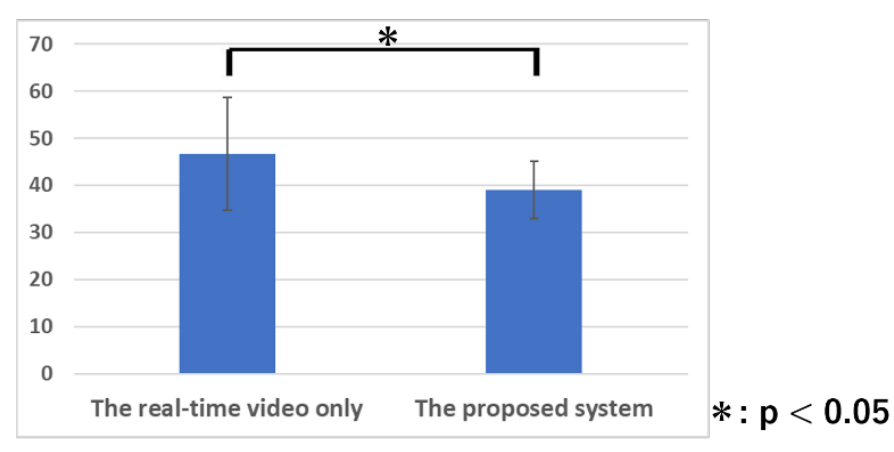

Fig. 8 Operation time (Subject2)

\subsection{Discussion}

Fig. 7 and Fig. 8 show that the proposed system is effective for the reduction of the operation time. The results of the t-test showed that the proposed system is statistically significant against the real-time video system for each subject. The reason for the difference in the significant trend between the subjects is lack of practice.

On the other hand, the average number of collisions was reduced. Therefore, it is considered that the proposed system shows effective views to help the operation.

Moreover, there are some feedbacks that they are effective in the questionnaire. However, the images that are found to be effective or ineffective are different for each subject.

\section{Conclusion}

The purpose of this study is to reduce the operation time and risk of collisions when the power shovel was remotely operated to sort slags. In the previous paper, the efficacy of the proposed system was verified in the real environment. In this paper, we carried out an additional experiment to get confirmation of the effectiveness of the proposed system.

Experiments showed that the proposed system was effective for the operation and it was statistically significant for subjects. Furthermore, the superiority of the proposed system is also confirmed by subjective evaluation.

In the future, it is necessary to increase the number of subjects and conduct a between-subjects design.

\section{References}

1. T. Toyota, Development of the Rubber Artificial Muscle Drive Type Remote Operation Robot for Disaster Restoration, Journal of the Robotics Society of Japan.Vol.33, 2015, pp. 600-606.

2. N. Shiroma et al. 13th IEEE International Workshop on Robot and Human Interactive Communication. 2004, pp. 107-112.

3. N. Shiroma et al. Transaction of the Society of Instrument and Control Engineers. Vol. 41, No. 12, 2005, pp. 10361043

4. A. Fukuda et al. Virtual bird's-eye view for remote operation of unmanned construction machinery, Reiwa $2^{\text {nd }}$ year Tokai-Section Joint Conference on Electrical, Electronics, Information, and Related Engineering, 2020. 